\title{
MESHLESS LOCAL RADIAL POINT INTERPOLATION (MLRPI) FOR GENERALIZED TELEGRAPH AND HEAT DIFFUSION EQUATION WITH NON-LOCAL BOUNDARY CONDITIONS
}

\author{
Elyas Shivanian, Arman Khodayari \\ Imam Khomeini International University, Department of Mathematics, Qazvin, Iran \\ e-mail address: shivanian@sci.ikiu.ac.ir
}

\begin{abstract}
In this paper, the meshless local radial point interpolation (MLRPI) method is formulated to the generalized one-dimensional linear telegraph and heat diffusion equation with non-local boundary conditions. The MLRPI method is categorized under meshless methods in which any background integration cells are not required, so that all integrations are carried out locally over small quadrature domains of regular shapes, such as lines in one dimensions, circles or squares in two dimensions and spheres or cubes in three dimensions. A technique based on the radial point interpolation is adopted to construct shape functions, also called basis functions, using the radial basis functions. These shape functions have delta function property in the frame work of interpolation, therefore they convince us to impose boundary conditions directly. The time derivatives are approximated by the finite difference time-stepping method. We also apply Simpson's integration rule to treat the non-local boundary conditions. Convergency and stability of the MLRPI method are clarified by surveying some numerical experiments.
\end{abstract}

Keyword: non-local boundary condition, meshless local radial point interpolation (MLRPI) method, local weak formulation, radial basis function, telegraph equation

\section{Introduction}

The telegraph equation is one of the important equations of mathematical physics with applications to many different fields such as transmission and propagation of electrical signals (Gonzalez-Velasco, 1995; Jordan and Puri, 1999), vibrational systems (Boyce and DiPrima, 1977), random walk theory (Banasiak and Mika, 1998) and mechanical systems (Tikhonov and Samarskii, 1990), etc. The heat diffusion and wave propagation equations are particular cases of the telegraph equation. Recently, increasing attention has been paid to the development, analysis and implementation of stable methods for numerical solutions of second-order hyperbolic equations. There have been many numerical methods for hyperbolic equations, such as the finite difference, the finite element, and the collocation methods, etc. (see Almenar et al., 1997; Ciment and Leventhal, 1978) and literatures therein.

On the other hand, many of natural phenomena in science and engineering have been modelled by non-local boundary value problems. In these non-local problems, some integral terms often appear in the boundary conditions. These types of problems constitute a special class of boundary value problems which widely appear in mathematical modelling of various processes of physics, heat transfer, ecology, thermoelasticity, chemistry, biology and industry.

According to the numerical results obtained, the present methods can be considered as practical and effective numerical techniques to solve telegraph equations with non-local boundary conditions. 
Let $\Omega=[0,1]$. Consider the 1D linear telegraph equation

$$
\frac{\partial^{2} u}{\partial t^{2}}+c \frac{\partial u}{\partial t}+b u-p \frac{\partial^{2} u}{\partial x^{2}}=f(x, t) \quad(x, t) \in \Omega \times[0, T]
$$

with the initial and non-local boundary conditions

$$
\begin{aligned}
& u(x, 0)=u_{0}(x) \quad \frac{\partial u}{\partial t}(x, 0)=\psi(x) \\
& u(0, t)=\gamma_{1} \int_{0}^{1} u(x, t) d x+\mu_{1}(t) \\
& u(1, t)=\gamma_{2} \int_{0}^{1} u(x, t) d x+\mu_{2}(t)
\end{aligned}
$$

where $c, b$ and $p$ are positive constants, $\gamma_{1}$ and $\gamma_{2}$ are constants and the functions $f, \psi, \mu_{1}(t)$ and $\mu_{2}(t)$ are assumed to be sufficiently smooth. Many partial differential equations are too complex to be solved by analytical methods. This caused mathematicians and engineers to come up with numerical methods such as the finite difference method (FDM) and the finite element method (FEM) to solve the equations. Although, these methods have been successfully applied to computational fluid dynamics problems, their accuracy depends critically on mesh quality and they have many difficulties in dealing with some complex problems. These difficulties can be overcome by meshless methods which have attracted considerable interest over the past few years (Kochmann and Venturini, 2014; Liu and Gu, 2005; Pan and Yuan, 2009; Sladek et al., 2006). These meshless methods do not require mesh for discretisation of the problem domain, and they construct approximate functions only via a set of nodes, so-called field nodes. In general, the meshless methods can be grouped into two categories. The first category is based on weak forms such as the element free Galerkin (EFG) method (Belytschko et al., 1995; Singh et al., 2007), the second category is based on strong forms such as meshless methods based on the radial basis functions (RBFs) (Dehghan and Shokri, 2008; Kansa, 1990). In addition, a meshless method based on combination of the strong and weak form has also been developed and is known as the meshless weak strong (MWS) form method. Due to the ill-conditioning of the resultant linear systems in the RBF-collocation method, various approaches are proposed to circumvent this problem (Libre et al., 2008; Ling and Schaback, 2008), being among them. The weak forms are used to derive a set of algebraic equations through a numerical integration process using a set of quadrature domain that may be constructed globally or locally in the domain of the problem. In the global formulation, background cells are required for the integration of the weak form. Strictly speaking, these meshless methods are not truly meshless methods. But in methods based on the local weak form formulation, numerical integrations are carried out over a local quadrature domains, therefore, no cells are required. As a result, they are referred to as truly meshless methods such as the meshless local Petrov-Galerkin (MLPG) method (Atluri and Zhu, 1998; Dehghan and Mirzaei, 2008; Shirzadi, 2014; Shivanian, 2015b). In the literature, several meshless weak form methods have been proposed such as the diffuse element method (DEM) (Nayroles et al., 1992), smooth particle hydrodynamic (SPH) (Bratsos, 2008; Dashtimanesh and Ghadimi, 2013), reproducing kernel particle method (RKPM) (Liu et al., 1995), boundary node method (BNM) (Mukherjee and Mukherjee, 1997), partition of unity finite element method (PUFEM) (Melenh and Babuska, 1996), finite sphere method (FSM) (De and Bathe, 2000), boundary point interpolation method (BPIM) (Gu and Liu, 2002) and boundary radial point interpolation method (BRPIM) (Gu and Liu, 2003). Liu applied the concept of MLPG and developed the meshless local radial point interpolation (MLRPI) method (Hosseini et al., 2015; Liu and Gu, 2001; Shivanian, 2013, 2015a; Shivanian and khodabandehlo, 2014). In this paper, 
we concentrate on the numerical solution of Eqs. (1.1) and (1.2) using the meshless local radial point interpolation (MLRPI) method. Besides, we use Simpson's integration rule to impose the non-local boundary condition.

\section{Approximation of field variables using the radial point interpolation method}

Consider a continuous function $u(x)$ defined in a domain $\Omega$, which is represented by a set of field nodes. The $u(x)$ at the point of interest $x$ is approximated as follows

$$
u(\mathbf{x})=\sum_{i=1}^{n} R_{i}(\mathbf{x}) a_{i}+\sum_{j=1}^{m} p_{j}(\mathbf{x}) b_{j}=\mathbf{R}^{\mathrm{T}}(\mathbf{x}) \mathbf{a}+\mathbf{P}^{\mathrm{T}}(\mathbf{x}) \mathbf{b}
$$

where $R_{i}(\mathbf{x})$ is thea radial basis function (RBF), $n$ is the number of RBFs, $p_{j}(\mathbf{x})$ is the monomial in the 1-D space $x$ and $m$ is the number of the monomials. In the present work, we have applied thin plate spline (TPS) multiquadrics (MQ) as the radial basis functions in Eq. (2.1). In order to determine $a_{i}$ and $b_{j}$ in Eq. (2.1), a support domain is needed for the point of interest at $x$ so that $n$ field nodes are included in the support domain. Then, coefficients $a_{i}$ and $b_{j}$ in Eq. (2.1) can be determined by the following system of $n$ linear equations

$$
\mathbf{U}_{s}=\mathbf{R}_{n} \mathbf{a}+\mathbf{P}_{m} \mathbf{b}
$$

in which the vector $U_{s}$ is

$$
\mathbf{U}_{s}=\left\{u_{1}, u_{2}, u_{3}, \ldots, u_{n}\right\}^{\mathrm{T}}
$$

moreover, $\mathbf{R}_{n}$ and $\mathbf{P}_{m}$ are the RBFs and polynomial moment matrices, respectively. On the other hand, Eq. (2.1) can be rewritten as

$$
u(\mathbf{x})=\mathbf{R}^{\mathrm{T}}(\mathbf{x}) \mathbf{a}+\mathbf{P}^{\mathrm{T}}(\mathbf{x}) \mathbf{b}=\left\{\mathbf{R}^{\mathrm{T}}(\mathbf{x}), \mathbf{P}^{\mathrm{T}}(\mathbf{x})\right\}\left[\begin{array}{l}
\mathbf{a} \\
\mathbf{b}
\end{array}\right]
$$

and then, by using that, we obtain

$$
\begin{aligned}
u(\mathbf{x}) & =\left\{\mathbf{R}^{\mathrm{T}}(\mathbf{x}), \mathbf{P}^{\mathrm{T}}(\mathbf{x})\right\}\left[\begin{array}{cc}
\mathbf{R}_{n} & \mathbf{P}_{m} \\
\mathbf{P}_{m}^{\mathrm{T}} & \mathbf{0}
\end{array}\right]^{-1} \widetilde{\mathbf{U}}_{s} \\
& =\left\{\mathbf{R}^{\mathrm{T}}(\mathbf{x}), \mathbf{P}^{\mathrm{T}}(\mathbf{x})\right\} \mathbf{G}^{-1} \widetilde{\mathbf{U}}_{s}=\widetilde{\boldsymbol{\Phi}}^{\mathrm{T}}(\mathbf{x}) \widetilde{\mathbf{U}}_{s}
\end{aligned}
$$

where $\widetilde{\boldsymbol{\Phi}}^{\mathrm{T}}(\mathbf{x})$ can be be introduced by

$$
\widetilde{\boldsymbol{\Phi}}^{\mathrm{T}}(\mathbf{x})=\left\{\mathbf{R}^{\mathrm{T}}(\mathbf{x}), \mathbf{P}^{\mathrm{T}}(\mathbf{x})\right\} \mathbf{G}^{-1}=\left\{\phi_{1}(\mathbf{x}), \phi_{2}(\mathbf{x}), \ldots, \phi_{n}(\mathbf{x}), \phi_{n+1}(\mathbf{x}), \ldots, \phi_{n+m}(\mathbf{x})\right\}
$$

The first $n$ functions of the above vector function are called the RPIM shape functions corresponding to the nodal displacements. We show them by the vector $\widetilde{\mathbf{\Phi}}^{\mathrm{T}}(\mathbf{x})$, so that it is

$$
\widetilde{\boldsymbol{\Phi}}^{\mathrm{T}}(\mathbf{x})=\left\{\phi_{1}(\mathbf{x}), \phi_{2}(\mathbf{x}), \ldots, \phi_{n}(\mathbf{x})\right\}
$$

Equation (2.5) is then transformed into

$$
u(\mathbf{x})=\widetilde{\mathbf{\Phi}}^{\mathrm{T}}(\mathbf{x}) \mathbf{U}_{s}=\sum_{i=1}^{n} \phi_{i}(\mathbf{x}) u_{i}
$$




\section{Finite differences approximation}

The following finite difference approximations of the order $O(\Delta t)^{2}$ are used for time discretization

$$
\begin{aligned}
& \frac{\partial^{2} u(\mathbf{x}, t)}{\partial t^{2}} \cong \frac{1}{\Delta t^{2}}\left(u^{(k+1)}(\mathbf{x})-2 u^{(k)}(\mathbf{x})+u^{(k-1)}(\mathbf{x})\right) \\
& \frac{\partial u(\mathbf{x}, t)}{\partial t} \cong \frac{1}{2 \Delta t}\left(u^{(k+1)}(\mathbf{x})-u^{(k-1)}(\mathbf{x})\right)
\end{aligned}
$$

Also, we employ the following approximation using the Crank-Nicolson technique

$$
\begin{aligned}
& u(\mathbf{x}, t) \cong \frac{1}{3}\left(u^{(k+1)}(\mathbf{x})+u^{(k)}(\mathbf{x})+u^{(k-1)}(\mathbf{x})\right) \\
& \frac{\partial^{2} u(\mathbf{x}, t)}{\partial \mathbf{x}^{2}} \cong \frac{1}{3}\left(\frac{\partial^{2} u^{(k+1)}(\mathbf{x}, t)}{\partial \mathbf{x}^{2}}+\frac{\partial^{2} u^{(k)}(\mathbf{x}, t)}{\partial \mathbf{x}^{2}}+\frac{\partial^{2} u^{(k-1)}(\mathbf{x}, t)}{\partial \mathbf{x}^{2}}\right)
\end{aligned}
$$

where $u^{k}(\mathbf{x})=u(\mathbf{x}, k \Delta t)$.

Using the above approximations, Eq. (1.1) can be written as

$$
\begin{aligned}
& \frac{1}{\Delta t^{2}}\left(u^{(k+1)}(\mathbf{x})-2 u^{(k)}(\mathbf{x})+u^{(k-1)}(\mathbf{x})\right)+\frac{c}{2 \Delta t}\left(u^{(k+1)}(\mathbf{x})-u^{(k-1)}(\mathbf{x})\right) \\
& \quad+\frac{b}{3}\left(u^{(k+1)}(\mathbf{x})+u^{(k)}(\mathbf{x})+u^{(k-1)}(\mathbf{x})\right) \\
& \quad-\frac{p}{3}\left(\frac{\partial^{2} u^{(k+1)}(\mathbf{x})}{\partial \mathbf{x}^{2}}+\frac{\partial^{2} u^{(k)}(\mathbf{x})}{\partial \mathbf{x}^{2}}+\frac{\partial^{2} u^{(k-1)}(\mathbf{x})}{\partial \mathbf{x}^{2}}\right)=\frac{1}{3}\left(f^{(k+1)}(\mathbf{x})+f^{(k)}(\mathbf{x})+f^{(k-1)}(\mathbf{x})\right)
\end{aligned}
$$

Supposing the notations $\lambda=1 / \Delta t^{2}$ and $\mu=c /(2 \Delta t)$, we obtain

$$
\begin{aligned}
(\lambda & \left.+\mu+\frac{b}{3}\right) u^{(k+1)}-\frac{p}{3} \frac{\partial^{2} u^{(k+1)}(\mathbf{x})}{\partial \mathbf{x}^{2}}=\left(2 \lambda-\frac{b}{3}\right) u^{(k)}+\frac{p}{3} \frac{\partial^{2} u^{(k)}(\mathbf{x})}{\partial \mathbf{x}^{2}} \\
& +\left(-\lambda+\mu-\frac{b}{3}\right) u^{(k-1)}+\frac{p}{3} \frac{\partial^{2} u^{(k-1)}(\mathbf{x})}{\partial \mathbf{x}^{2}}+\frac{1}{3}\left(f^{(k+1)}(\mathbf{x})+f^{(k)}(\mathbf{x})+f^{(k-1)}(\mathbf{x})\right) .
\end{aligned}
$$

\section{The meshless local weak form formulation}

Instead of setting the global weak form, the MLRPI method sets up the weak form over the local quadrature cell such as $\Omega_{q}$, which is a small region taken for each node in the global domain $\Omega$. The local quadrature cells overlap with each other and cover the whole global domain $\Omega$. The local quadrature cells could be of any geometric shape and size. In one dimensional problems, they are lines (intervals). The local weak form of Eq. (3.4) for $\mathbf{x}_{i} \in \Omega_{q}^{i}=\left(x_{i}-r_{q}, x_{i}+r_{q}\right)$ can be constructed as

$$
\begin{aligned}
& \int_{\Omega_{q}^{i}}\left[\left(\left(\lambda+\mu+\frac{b}{3}\right) u^{(k+1)}-\frac{p}{3} \frac{\partial^{2} u^{(k+1)}(\mathbf{x})}{\partial \mathbf{x}^{2}}\right] \nu(\mathbf{x}) d x=\int_{\Omega_{q}^{i}}\left[\left(2 \lambda-\frac{b}{3}\right) u^{(k)}+\frac{p}{3} \frac{\partial^{2} u^{(k)}(\mathbf{x})}{\partial \mathbf{x}^{2}}\right] \nu(\mathbf{x}) d x\right. \\
& \quad+\int_{\Omega_{q}^{i}}\left[\left(\left(-\lambda+\mu-\frac{b}{3}\right) u^{(k-1)}+\frac{p}{3} \frac{\partial^{2} u^{(k-1)}(\mathbf{x})}{\partial \mathbf{x}^{2}}\right] \nu(\mathbf{x}) d x\right. \\
& \quad+\int_{\Omega_{q}^{i}}\left[\frac{1}{3}\left(f^{(k+1)}(\mathbf{x})+f^{(k)}(\mathbf{x})+f^{(k-1)}(\mathbf{x})\right)\right] \nu(\mathbf{x}) d x
\end{aligned}
$$


where $\Omega_{q}^{i}$ is the local quadrature domain corresponding to the point $i$, and $\nu(\mathbf{x})$ is the Heaviside step function defined by (Hu et al., 2006; Liu et al., 2006)

$$
\nu(\mathbf{x})= \begin{cases}1 & x \in \Omega_{q} \\ 0 & x \notin \Omega_{q}\end{cases}
$$

as the test function in each local quadrature domain. Hence, we obtain

$$
\begin{aligned}
(\lambda & \left.+\mu+\frac{b}{3}\right) \int_{\Omega_{q}^{i}} u^{(k+1)} \nu(\mathbf{x}) d x-\frac{p}{3} \int_{\Omega_{q}^{i}} \frac{\partial^{2} u^{(k+1)}(\mathbf{x})}{\partial \mathbf{x}^{2}} \nu(\mathbf{x}) d x \\
& =\left(2 \lambda-\frac{b}{3}\right) \int_{\Omega_{q}^{i}} u^{(k)} \nu(\mathbf{x}) d x+\frac{p}{3} \int_{\Omega_{q}^{i}} \frac{\partial^{2} u^{(k)}(\mathbf{x})}{\partial \mathbf{x}^{2}} \nu(\mathbf{x}) d x \\
& +\left(-\lambda+\mu-\frac{b}{3}\right) \int_{\Omega_{q}^{i}} u^{(k-1)} \nu(\mathbf{x}) d x+\frac{p}{3} \int_{\Omega_{q}^{i}} \frac{\partial^{2} u^{(k-1)}(\mathbf{x})}{\partial \mathbf{x}^{2}} \nu(\mathbf{x}) d x \\
& +\frac{1}{3} \int_{\Omega_{q}^{i}}\left(f^{(k+1)}(\mathbf{x})+f^{(k)}(\mathbf{x})+f^{(k-1)}(\mathbf{x})\right) \nu(\mathbf{x}) d x
\end{aligned}
$$

Using integration by parts, one obtains

$$
\int_{\Omega_{q}^{i}} \frac{\partial^{2} u^{(k)}(\mathbf{x})}{\partial \mathbf{x}^{2}} \nu(\mathbf{x}) d x=\left.\nu(\mathbf{x}) \frac{\partial u^{(k)}(\mathbf{x})}{\partial \mathbf{x}}\right|_{\mathbf{x}=x_{i}-r_{q}} ^{\mathbf{x}=x_{i}+r_{q}}-\int_{\Omega_{q}^{i}} \frac{\partial u^{(k)}(\mathbf{x})}{\partial \mathbf{x}} \frac{\partial \nu(\mathbf{x})}{\partial x} d x
$$

Then, by applying the test function, the following local weak equation is obtained

$$
\begin{aligned}
(\lambda & \left.+\mu+\frac{b}{3}\right) \int_{\Omega_{q}^{i}} u^{(k+1)} d x-\frac{p}{3}\left(\left.\frac{\partial u^{(k+1)}(\mathbf{x})}{\partial \mathbf{x}}\right|_{\mathbf{x}=x_{i}-r_{q}} ^{\mathbf{x}=x_{i}+r_{q}}\right) \\
& =\left(2 \lambda-\frac{b}{3}\right) \int_{\Omega_{q}^{i}} u^{(k)} d x+\frac{p}{3}\left(\left.\frac{\partial u^{(k)}(\mathbf{x})}{\partial \mathbf{x}}\right|_{\mathbf{x}=x_{i}-r_{q}} ^{\mathbf{x}=x_{i}+r_{q}}\right) \\
& +\left(-\lambda+\mu-\frac{b}{3}\right) \int_{\Omega_{q}^{i}} u^{(k-1)} d x+\frac{p}{3}\left(\left.\frac{\partial u^{(k-1)}(\mathbf{x})}{\partial \mathbf{x}}\right|_{\mathbf{x}=x_{i}-r_{q}} ^{\mathbf{x}=x_{i}+r_{q}}\right) \\
& +\frac{1}{3} \int_{\Omega_{q}^{i}}\left(f^{(k+1)}(\mathbf{x})+f^{(k)}(\mathbf{x})+f^{(k-1)}(\mathbf{x})\right) d x
\end{aligned}
$$

\section{Discretization in the MLRPI method}

In this Section, we consider Eq. (4.5) to see how to obtain discrete equations. Consider $N$ regularly located points on the boundary and domain of the problem, i.e. interval $[0,1]$, so that the distance between two consecutive nodes in each direction is constant and equal to $h$. Assuming that $u\left(\mathbf{x}_{i}, k \Delta t\right), i=1,2, \ldots, N$ are known, our aim is to compute $u\left(\mathbf{x}_{i},(k+1) \Delta t\right)$, $i=1,2, \ldots, N$. So, we have $N$ unknowns and to compute these unknowns, we need $N$ equations. To obtain the discrete equations from locally weak forms (4.5) for the nodes located in the interior of the domain, i.e., for $\mathbf{x}_{i} \in$ interior $\Omega$, we substitute approximation formulas (2.8) into local integral equations (4.5) to have 


$$
\begin{aligned}
& {\left[\left(\lambda+\mu+\frac{b}{3}\right) \sum_{j=1}^{N}\left(\int_{\Omega_{q}^{i}} \phi_{j}(\mathbf{x}) d x\right)-\frac{p}{3} \sum_{j=1}^{N}\left(\left.\frac{\partial \phi_{j}(\mathbf{x})}{\partial \mathbf{x}}\right|_{\mathbf{x}=x_{i}+r_{q}}-\left.\frac{\partial \phi_{j}(\mathbf{x})}{\partial \mathbf{x}}\right|_{\mathbf{x}=x_{i}-r_{q}}\right)\right] u_{j}^{(k+1)}} \\
& \quad=\left[\left(2 \lambda-\frac{b}{3}\right) \sum_{j=1}^{N}\left(\int_{\Omega_{q}^{i}} \phi_{j}(\mathbf{x}) d x\right)+\frac{p}{3} \sum_{j=1}^{N}\left(\left.\frac{\partial \phi_{j}(\mathbf{x})}{\partial \mathbf{x}}\right|_{\mathbf{x}=x_{i}+r_{q}}-\left.\frac{\partial \phi_{j}(\mathbf{x})}{\partial \mathbf{x}}\right|_{\mathbf{x}=x_{i}-r_{q}}\right)\right] u_{j}^{(k)} \\
& \quad+\left[\left(-\lambda+\mu-\frac{b}{3}\right) \sum_{j=1}^{N}\left(\int_{\Omega_{q}^{i}} \phi_{j}(\mathbf{x}) d x\right)+\frac{p}{3} \sum_{j=1}^{N}\left(\left.\frac{\partial \phi_{j}(\mathbf{x})}{\partial \mathbf{x}}\right|_{\mathbf{x}=x_{i}+r_{q}}-\left.\frac{\partial \phi_{j}(\mathbf{x})}{\partial \mathbf{x}}\right|_{\mathbf{x}=x_{i}-r_{q}}\right)\right] u_{j}^{(k-1)} \\
& \quad+\frac{1}{3} \int_{\Omega_{q}^{i}}\left(f^{(k+1)}(\mathbf{x})+f^{(k)}(\mathbf{x})+f^{(k-1)}(\mathbf{x})\right) d x
\end{aligned}
$$

\section{Numerical implementation of the MLRPI method}

By using Simpson's integration rule for nodes which are located on the boundary, we have for all $k$

$$
\begin{aligned}
& u^{(k)}\left(x_{1}\right)=\gamma_{1} \frac{h}{3}\left[u^{(k)}\left(x_{1}\right)+4 u^{(k)}\left(x_{2}\right)+2 u^{(k)}\left(x_{3}\right)+\ldots+4 u^{(k)}\left(x_{N-1}\right)+u^{(k)}\left(x_{N}\right)\right]+\mu_{1}(k \Delta t) \\
& u^{(k)}\left(x_{N}\right)=\gamma_{2} \frac{h}{3}\left[u^{(k)}\left(x_{1}\right)+4 u^{(k)}\left(x_{2}\right)+2 u^{(k)}\left(x_{3}\right)+\ldots+4 u^{(k)}\left(x_{N-1}\right)+u^{(k)}\left(x_{N}\right)\right]+\mu_{2}(k \Delta t)
\end{aligned}
$$

where $x_{1}=0$ and $x_{N}=1$.

The matrix forms of Eqs. (5.1) and (6.1) for all $N$ nodal points in the domain and the boundary of the problem are given below

$$
\begin{aligned}
& {\left[\left(\lambda+\mu+\frac{b}{3}\right) \sum_{j=1}^{N} A_{i, j}-\frac{p}{3} \sum_{j=1}^{N} B_{i, j}\right] u_{j}^{(k+1)}=\left[\left(2 \lambda-\frac{b}{3}\right) \sum_{j=1}^{N} A_{i, j}+\frac{p}{3} \sum_{j=1}^{N} B_{i, j}\right] u_{j}^{(k)}} \\
& +\left[\left(-\lambda+\mu-\frac{b}{3}\right) \sum_{j=1}^{N} A_{i, j}+\frac{p}{3} \sum_{j=1}^{N} B_{i, j}\right] u_{j}^{(k-1)}+E_{i}(k-1, k, k+1)
\end{aligned}
$$

where

$$
\begin{aligned}
& A_{i, j}=\int_{\Omega_{q}^{i}} \phi_{j}(\mathbf{x}) d x \\
& B_{i, j}=\left(\left.\frac{\partial \phi_{j}(\mathbf{x})}{\partial \mathbf{x}}\right|_{\mathbf{x}=x_{i}+r_{q}}-\left.\frac{\partial \phi_{j}(\mathbf{x})}{\partial \mathbf{x}}\right|_{\mathbf{x}=x_{i}-r_{q}}\right) \\
& E_{i}(k-1, k, k+1)=\frac{1}{3} \int_{\Omega_{q}^{i}}\left(f^{(k+1)}(\mathbf{x})+f^{(k)}(\mathbf{x})+f^{(k-1)}(\mathbf{x})\right) d x
\end{aligned}
$$

Assuming

$$
\begin{array}{ll}
\mathbf{A}_{i, j}=\left(\lambda+\mu+\frac{b}{3}\right) A_{i, j}-\frac{p}{3} B_{i, j} & \mathbf{B}_{i, j}=\left(2 \lambda-\frac{b}{3}\right) A_{i, j}+\frac{p}{3} B_{i, j} \\
\mathbf{C}_{i, j}=\left(-\lambda+\mu-\frac{b}{3}\right) A_{i, j}+\frac{p}{3} B_{i, j} & \mathbf{U}=\left\{u_{i}\right\}_{N \times 1} \\
\mathbf{E}^{k}=\left[E_{1}(k-1, k, k+1), E_{2}(k-1, k, k+1), \ldots, E_{N}(k-1, k, k+1)\right]^{\mathrm{T}}
\end{array}
$$

yields 


$$
\mathbf{A} \mathbf{U}^{(k+1)}=\mathbf{B} \mathbf{U}^{(k)}+\mathbf{C} \mathbf{U}^{(k-1)}+\mathbf{E}^{k}
$$

Furthermore, to satisfy Eqs. (6.1), for both nodes belong to the boundary, i.e., $\left\{x_{1}, x_{N}\right\}$, we set

$$
\begin{aligned}
& \mathbf{E}_{i}^{k}= \begin{cases}\mu_{1}(k \Delta t) & i=1 \\
\mu_{2}(k \Delta t) & i=N\end{cases} \\
& \forall j: \quad \mathbf{B}_{i, j}=\mathbf{C}_{i, j}=\mathbf{0} \quad i=1, N \\
& \mathbf{A}_{1}=\left[1-\gamma_{1} \frac{h}{3},-4 \gamma_{1} \frac{h}{3},-2 \gamma_{1} \frac{h}{3}, \ldots,-4 \gamma_{1} \frac{h}{3},-\gamma_{1} \frac{h}{3}\right] \\
& \mathbf{A}_{N}=\left[-\gamma_{2} \frac{h}{3},-4 \gamma_{2} \frac{h}{3},-2 \gamma_{2} \frac{h}{3}, \ldots,-4 \gamma_{2} \frac{h}{3}, 1-\gamma_{2} \frac{h}{3}\right]
\end{aligned}
$$

where $\mathbf{A}_{1}$ and $\mathbf{A}_{N}$ are the first and $N$-th rows of the matrix $\mathbf{A}$, respectively.

At the first time level, when $n=0$, according to the initial conditions that are introduced in Eq. (1.2), we apply the following assumptions

$$
u^{(0)}=u_{0} \quad u^{(-1)} \cong u^{(1)}-2 \Delta t \psi(x)
$$

where

$$
u_{0}=\left[u_{0}\left(x_{1}\right), u_{0}\left(x_{2}\right), \ldots, u_{0}\left(x_{N}\right)\right]^{\mathrm{T}} \quad \psi=\left[\psi\left(x_{1}\right), \psi\left(x_{2}\right), \ldots, \psi\left(x_{N}\right)\right]^{\mathrm{T}}
$$

\section{Numerical experiments}

In this Section, two numerical expriments for application of the meshless local radial point interpolation method (MLRPI) in solving the one-dimensional linear telegraph equation with non-local boundary conditions are presented. In both examples, the domain integrals are evaluated with 3 points Gaussian quadrature rule. In these problems, the regular distributed nodal points are used. Also, in order to implement the meshless local weak form in these cases, the radius of the local quadrature domain $r_{q}=0.8 h$ is selected, where $h$ is the distance between the nodes in the $x$ direction $(h=\Delta x)$. The size of $r_{q}$ is such that the union of these sub-domains must cover the whole global domain. The radius of the support domain to the local radial point interpolation method is $r_{s}=4 r_{q}$. This size is significant enough to have a sufficient number of nodes $(n)$ to give appropriate shape functions. Also, in Eq. (2.1), we set $m=5$.

Example 1. We set $c=20, b=25$ and $p=1$. The exact solution of the first example is taken as $u(x, t)=t^{3}\left(2 x^{3}-x+4\right),(x, t) \in[0,1] \times[0,1]$. According to this exact solution, $f(x, t)$ is given by

$$
f(x, t)=\left(6 t+3 c t^{2}\right)\left(2 x^{3}-x+4\right)+b t^{3}\left(2 x^{3}-x+4\right)-12 p x t^{3}
$$

the initial conditions are

$$
u(x, 0)=0 \quad \frac{\partial u}{\partial t}(x, 0)=0
$$

and the non-local boundary conditions take the form

$$
\begin{aligned}
u(0, t) & =\int_{0}^{1} u(x, t) d x & t \geqslant 0 \\
u(1, t) & =\frac{5}{4} \int_{0}^{1} u(x, t) d x & t \geqslant 0
\end{aligned}
$$


Tables 1 and 2 as well as Fig. 1a show the results of the MLRPI method to solve Example 1 using TPS as the radial basis function. Also, Tables 3 and 4 as well as Fig. 1b illustrate the results of the current method to solve Example 1 using MQ as the radial basis function. As it is seen, the MLRPI method is of high accuracy. Furthermore, it is seen that the method is convergent with respect to the spatial and time variable using both TPS and MQ.
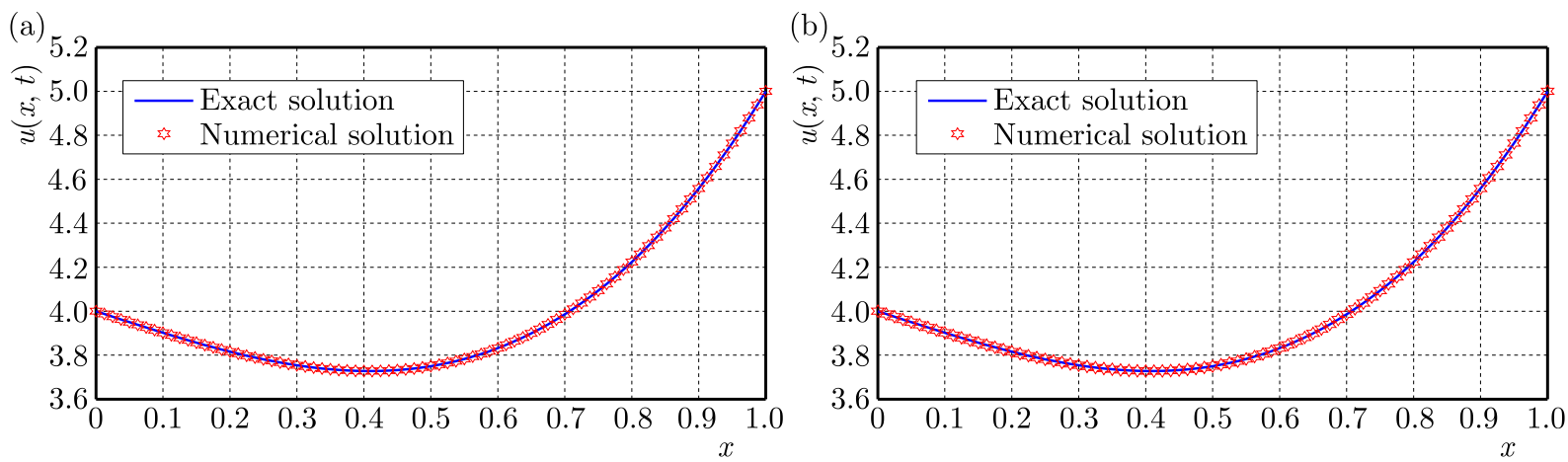

Fig. 1. Numerical solutions and the exact solution at time $t=1.0$ for Example 1: (a) using TPS,

(b) using MQ. The solid line corresponds to the exact solution, the starred line corresponds to the numerical solution of the MLRPI with $\Delta t=0.0001$ and $\Delta x=0.0125$

Table 1. The $L^{1}, L^{2}$ and $L^{\infty}$ errors calculated by MLRPI for Example 1 with different $\Delta x$ and $\Delta t$ at time $t=1.0$ (using TPS)

\begin{tabular}{|c|c|c|c|c|}
\hline$\Delta t$ & $\Delta x$ & $\|E\|_{1}$ & $\|E\|_{2}$ & $\|E\|_{\infty}$ \\
\hline \hline 0.001 & 0.25 & $4.070835 \mathrm{E}-03$ & $2.136594 \mathrm{E}-03$ & $1.508984 \mathrm{E}-03$ \\
\hline 0.001 & 0.125 & $3.470766 \mathrm{E}-04$ & $1.792837 \mathrm{E}-04$ & $1.587210 \mathrm{E}-04$ \\
\hline 0.001 & 0.1 & $1.718355 \mathrm{E}-04$ & $7.995307 \mathrm{E}-05$ & $6.922815 \mathrm{E}-05$ \\
\hline 0.001 & 0.05 & $6.156571 \mathrm{E}-05$ & $1.401474 \mathrm{E}-05$ & $5.949385 \mathrm{E}-06$ \\
\hline
\end{tabular}

Table 2. The $L^{1}, L^{2}$ and $L^{\infty}$ errors calculated by MLRPI for Example 1 with different $\Delta x$ and $\Delta t$ at time $t=1.0$ (using TPS)

\begin{tabular}{|c|c|c|c|c|}
\hline$\Delta t$ & $\Delta x$ & $\|E\|_{1}$ & $\|E\|_{2}$ & $\|E\|_{\infty}$ \\
\hline \hline 0.001 & 0.0125 & $1.945099 \mathrm{E}-04$ & $2.169317 \mathrm{E}-05$ & $2.997031 \mathrm{E}-06$ \\
\hline 0.0005 & 0.0125 & $4.863614 \mathrm{E}-05$ & $5.424111 \mathrm{E}-06$ & $7.494035 \mathrm{E}-07$ \\
\hline 0.00025 & 0.0125 & $1.217382 \mathrm{E}-05$ & $1.357562 \mathrm{E}-06$ & $1.875924 \mathrm{E}-07$ \\
\hline 0.0001 & 0.0125 & $1.964876 \mathrm{E}-06$ & $2.192364 \mathrm{E}-07$ & $3.026972 \mathrm{E}-08$ \\
\hline
\end{tabular}

Table 3. The $L^{1}, L^{2}$ and $L^{\infty}$ errors calculated by MLRPI for Example 1 with different $\Delta x$ and $\Delta t$ at time $t=1.0$ (using MQ)

\begin{tabular}{|c|c|c|c|c|}
\hline$\Delta t$ & $\Delta x$ & $\|E\|_{1}$ & $\|E\|_{2}$ & $\|E\|_{\infty}$ \\
\hline \hline 0.001 & 0.25 & $4.070835 \mathrm{E}-03$ & $2.136594 \mathrm{E}-03$ & $1.508984 \mathrm{E}-03$ \\
\hline 0.001 & 0.125 & $3.459171 \mathrm{E}-04$ & $1.780828 \mathrm{E}-04$ & $1.575668 \mathrm{E}-04$ \\
\hline 0.001 & 0.1 & $1.715535 \mathrm{E}-04$ & $7.972770 \mathrm{E}-05$ & $6.898580 \mathrm{E}-05$ \\
\hline 0.001 & 0.05 & $6.154538 \mathrm{E}-05$ & $1.401788 \mathrm{E}-05$ & $5.974310 \mathrm{E}-06$ \\
\hline
\end{tabular}


Table 4. The $L^{1}, L^{2}$ and $L^{\infty}$ errors calculated by MLRPI for Example 1 with different $\Delta x$ and $\Delta t$ at time $t=1.0$ (using MQ)

\begin{tabular}{|c|c|c|c|c|}
\hline$\Delta t$ & $\Delta x$ & $\|E\|_{1}$ & $\|E\|_{2}$ & $\|E\|_{\infty}$ \\
\hline \hline 0.001 & 0.0125 & $1.942840 \mathrm{E}-04$ & $2.166659 \mathrm{E}-05$ & $2.993550 \mathrm{E}-06$ \\
\hline 0.0005 & 0.0125 & $4.841030 \mathrm{E}-05$ & $5.397581 \mathrm{E}-06$ & $7.459259 \mathrm{E}-07$ \\
\hline 0.00025 & 0.0125 & $1.194834 \mathrm{E}-05$ & $1.331264 \mathrm{E}-06$ & $1.841192 \mathrm{E}-07$ \\
\hline 0.0001 & 0.0125 & $1.740619 \mathrm{E}-06$ & $1.944979 \mathrm{E}-07$ & $2.682201 \mathrm{E}-08$ \\
\hline
\end{tabular}

Example 2. We set $c=20, b=25$ and $p=1$. The exact solution of the this example is taken as $u(x, t)=t^{3} \sin (x+1),(x, t) \in[0,1] \times[0,1]$. According to this exact solution, $f(x, t)$ is given by

$$
f(x, t)=\left(6 t+3 c t^{2}\right) \sin (x+1)+b t^{3} \sin (x+1)+p t^{3} \sin (x+1)
$$

the initial conditions are

$$
u(x, 0)=0 \quad \frac{\partial u}{\partial t}(x, 0)=0
$$

and the non-local boundary conditions take the form

$$
\begin{array}{ll}
u(0, t)=0.8797864387 \int_{0}^{1} u(x, t) d x & t \geqslant 0 \\
u(1, t)=0.950701283 \int_{0}^{1} u(x, t) d x & t \geqslant 0
\end{array}
$$

Tables 5 and 6 as well as Fig. 2a show the results of the MLRPI method to solve Example 2 using TPS as the radial basis function. Besides, Tables 7 and 8 as well as Fig. 2b demonstrate the results of the present method to solve Example 2 using MQ as the radial basis function. As it is seen, the MLRPI method is of high accuracy. Moreover, we see that the convergence with respect to both the time step $(\Delta t)$ and the number of nodal points $(N)$ are hold, no matter which kind of RBF we use.
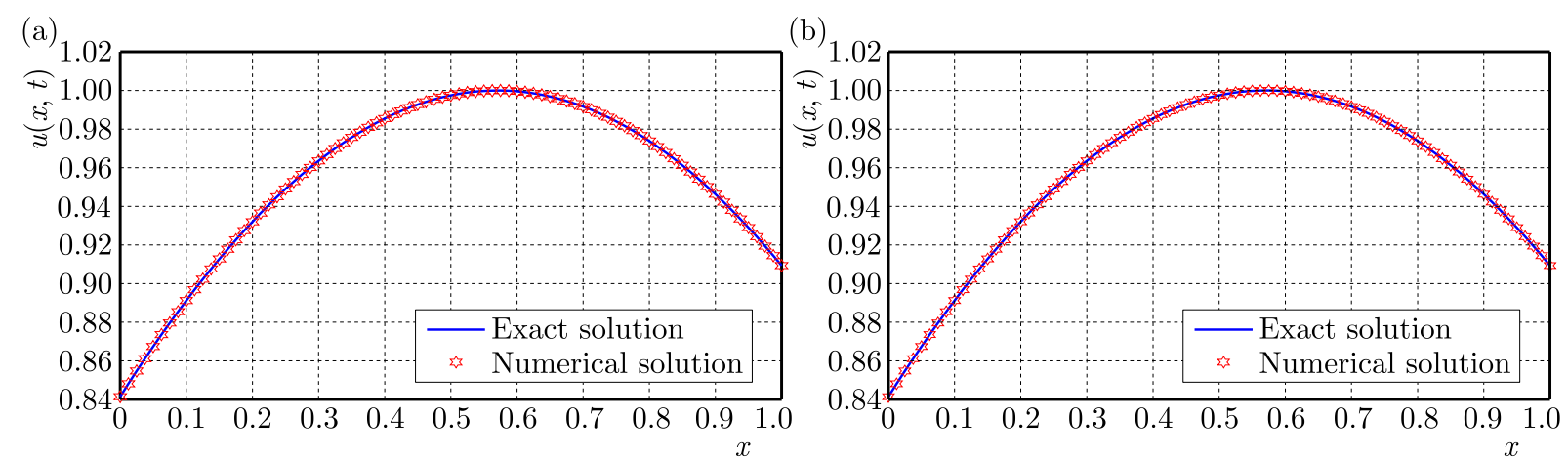

Fig. 2. Numerical solutions and the exact solution at time $t=1.0$ for Example 2: (a) using TPS, (b) using MQ. The solid line corresponds to the exact solution, the starred line corresponds to the numerical solution of the MLRPI with $\Delta t=0.0001$ and $\Delta x=0.0125$

On the top of that, the MLRPI method can be used to solve complex engineering problems with lower computational cost, higher accuracy, simpler construction of higher-order shape functions and easier handling of large deformation problems. 
Table 5. The $L^{1}, L^{2}$ and $L^{\infty}$ errors calculated by MLRPI for Example 2 with different $\Delta x$ and $\Delta t$ at time $t=1.0$ (using TPS)

\begin{tabular}{|c|c|c|c|c|}
\hline$\Delta t$ & $\Delta x$ & $\|E\|_{1}$ & $\|E\|_{2}$ & $\|E\|_{\infty}$ \\
\hline \hline 0.001 & 0.25 & $4.467206 \mathrm{E}-04$ & $2.263494 \mathrm{E}-04$ & $1.434185 \mathrm{E}-04$ \\
\hline 0.001 & 0.125 & $2.548359 \mathrm{E}-05$ & $1.304920 \mathrm{E}-05$ & $1.148951 \mathrm{E}-05$ \\
\hline 0.001 & 0.1 & $1.502825 \mathrm{E}-05$ & $6.430589 \mathrm{E}-06$ & $5.320887 \mathrm{E}-06$ \\
\hline 0.001 & 0.05 & $1.175112 \mathrm{E}-05$ & $2.629557 \mathrm{E}-06$ & $8.956239 \mathrm{E}-07$ \\
\hline
\end{tabular}

Table 6. The $L^{1}, L^{2}$ and $L^{\infty}$ errors calculated by MLRPI for Example 2 with different $\Delta x$ and $\Delta t$ at time $t=1.0$ (using TPS)

\begin{tabular}{|c|c|c|c|c|}
\hline$\Delta t$ & $\Delta x$ & $\|E\|_{1}$ & $\|E\|_{2}$ & $\|E\|_{\infty}$ \\
\hline 0.001 & 0.0125 & $4.418773 \mathrm{E}-05$ & $4.914641 \mathrm{E}-06$ & $5.709157 \mathrm{E}-07$ \\
\hline 0.0005 & 0.0125 & $1.104494 \mathrm{E}-05$ & $1.228475 \mathrm{E}-06$ & $1.426643 \mathrm{E}-07$ \\
\hline 0.00025 & 0.0125 & $2.7603974 \mathrm{E}-06$ & $3.070796 \mathrm{E}-07$ & $3.561701 \mathrm{E}-08$ \\
\hline 0.0001 & 0.0125 & $4.405399 \mathrm{E}-07$ & $4.920947 \mathrm{E}-08$ & $6.985131 \mathrm{E}-09$ \\
\hline
\end{tabular}

Table 7. The $L^{1}, L^{2}$ and $L^{\infty}$ errors calculated by MLRPI for Example 2 with different $\Delta x$ and $\Delta t$ at time $t=1.0$ (using $\mathrm{MQ}$ )

\begin{tabular}{|c|c|c|c|c|}
\hline$\Delta t$ & $\Delta x$ & $\|E\|_{1}$ & $\|E\|_{2}$ & $\|E\|_{\infty}$ \\
\hline 0.001 & 0.25 & $4.467206 \mathrm{E}-04$ & $2.263494 \mathrm{E}-04$ & $1.434185 \mathrm{E}-04$ \\
\hline 0.001 & 0.125 & $2.542164 \mathrm{E}-05$ & $1.296910 \mathrm{E}-05$ & $1.140565 \mathrm{E}-05$ \\
\hline 0.001 & 0.1 & $1.500617 \mathrm{E}-05$ & $6.414381 \mathrm{E}-06$ & $5.302278 \mathrm{E}-06$ \\
\hline 0.001 & 0.05 & $1.174746 \mathrm{E}-05$ & $2.629195 \mathrm{E}-06$ & $8.987330 \mathrm{E}-07$ \\
\hline
\end{tabular}

Table 8. The $L^{1}, L^{2}$ and $L^{\infty}$ errors calculated by MLRPI for Example 2 with different $\Delta x$ and $\Delta t$ at time $t=1.0$ (using MQ)

\begin{tabular}{|c|c|c|c|c|}
\hline$\Delta t$ & $\Delta x$ & $\|E\|_{1}$ & $\|E\|_{2}$ & $\|E\|_{\infty}$ \\
\hline 0.001 & 0.0125 & $4.413908 \mathrm{E}-05$ & $4.909225 \mathrm{E}-06$ & $5.701902 \mathrm{E}-07$ \\
\hline 0.0005 & 0.0125 & $1.099625 \mathrm{E}-05$ & $1.223055 \mathrm{E}-06$ & $1.419426 \mathrm{E}-07$ \\
\hline 0.00025 & 0.0125 & $2.711815 \mathrm{E}-06$ & $3.016704 \mathrm{E}-07$ & $3.548134 \mathrm{E}-08$ \\
\hline 0.0001 & 0.0125 & $3.928077 \mathrm{E}-07$ & $4.390304 \mathrm{E}-08$ & $5.957403 \mathrm{E}-09$ \\
\hline
\end{tabular}

\section{Conclusions}

In the aforementioned discussion, we applied the meshless local radial point interpolation (MLRPI) method to solve the linear telegraph equation with non-local boundary conditions. The radial point interpolation method is adopted for approximating the field variable. Also the weak form of the discretized equations has been constructed on local subdomains. So, this method requires neither domain element nor background cells in either the interpolation or the intergration. It means this method is a truly meshless method. Furthermore, time discretization has been done using finite difference techniques. The principal benefit of the method is to capture the behavior of the solution for similar problems with non-local boundary conditions where most of schemes fail. Also, the MLRPI method can easily handle the damage of the components, such as fracture which is very useful to simulate material breakage. Finally, accuracy and usefulness of the proposed method are illustrated by two examples. 


\section{Acknowledgments}

The authors are grateful to anonymous reviewers for carefully reading this paper and for their comments and suggestions which have improved the paper.

\section{References}

1. Almenar P., Jodar L., Martin J.A., 1997, Mixed problems for the time-dependent telegraph equation: Continuous numerical solutions with a priori error bounds, Mathematical and Computer Modelling, 25, 11, 31-44

2. Atluri S., Zhu T., 1998, A new meshless local Petrov-Galerkin (MLPG) approach in computational mechanics, Computational Mechanics, 22, 117-127

3. BAnAsiak J., Mika J.R., 1998, Singularly perturbed telegraph equations with applications in the random walk theory, Journal of Applied Mathematics and Stochastic Analysis, 11, 1, 9-28

4. Belytschko T., Lu Y.Y., Gu L., 1995, Element free Galerkin methods for static and dynamic fracture, International Journal of Solids and Structures, 32, 2547-2570

5. Boyce W.E., DiPrima R.C., 1977, Differential Equations Elementary and Boundary Value Problems, Wiley, New York

6. Bratsos A.G., 2008, An improved numerical scheme for the sine-Gordon equation in $2+1$ dimensions, International Journal for Numerical Methods in Engineering, 75, 787-799

7. Ciment M., Leventhal S.H., 1978, A note on the operator compact implicit method for the wave equation, Mathematics of Computation, 32, 143-147

8. Dashtimanesh A., Ghadimi P., 2013, A three-dimensional SPH model for detailed study of free surface deformation, just behind a rectangular planing hull, Journal of the Brazilian Society of Mechanical Sciences and Engineering, 35, 4, 369-380

9. De S., Bathe K.J., 2000, The method of finite spheres, Computational Mechanics, 25, 329-345

10. Dehghan M., Mirzaei D., 2008, The meshless local Petrov-Galerkin (MLPG) method for the generalized two-dimensional non-linear Schrödinger equation, Engineering Analysis with Boundary Elements, 32, 747-756

11. Dehghan M., Shokri A., 2008, A numerical method for solution of the two dimensional sineGordon equation using the radial basis functions, Mathematics and Computers in Simulation, 79, $700-715$

12. Gonzalez-Velasco E.A., 1995, Fourier Analysis and Boundary Value Problems, Academic Press, New York

13. Gu Y., LiU G., 2002, A boundary point interpolation method for stress analysis of solids, Computational Mechanics, 28, 47-54

14. GU Y.T., LiU G.R., 2003, A boundary radial point interpolation method (BRPIM) for 2-D structural analyses, Structural Engineering and Mechanics, 15, 535-550

15. Hosseini V.R., Shivanian E., Chen W., 2015, Local integration of 2-D fractional telegraph equation via local radial point interpolant approximation, European Physical Journal Plus, 130, $33-54$

16. Hu D., Long S., Liu K., Li G., 2006, A modified meshless local Petrov-Galerkin method to elasticity problems in computer modeling and simulation, Engineering Analysis with Boundary Elements, 30, 399-404

17. Jordan P.M., Puri A., 1999, Digital signal propagation in dispersive media, Journal of Applied Physics, 85, 3, 1273-1282

18. Kansa E., 1990, Multiquadrics-a scattered data approximation scheme with applications to computational fluid-dynamics. I. Surface approximations and partial derivative estimates, Computers and Mathematics with Applications, 19, 8/9, 127-145 
19. Kochmann D.M., Venturini G.N., 2014, A meshless quasicontinuum method based on local maximum-entropy interpolation, Modelling and Simulation in Materials Science and Engineering, 22, 3, 034007

20. Libre N., Emdadi A., Kansa E., Shekarchi M., Rahimian M., 2008, A fast adaptive wavelet scheme in RBF collocation for nearly singular potential PDEs, Computer Modeling in Engineering and Sciences, 38, 3, 263-284

21. Ling L., SCHABACK R., 2008, Stable and convergent unsymmetric meshless collocation methods, SIAM Journal of Numerical Analysis, 46, 3, 1097-1115

22. LiU G.R., GU Y.T., 2001, A local radial point interpolation method (LR-PIM) for free vibration analyses of 2-D solids, Journal of Sound and Vibration, 246, 1, 29-46

23. Liu G., Gu Y., 2005, An Introduction to Meshfree Methods and their Programing, Springer

24. Liu K., Long S., Li G., 2006, A simple and less-costly meshless local Petrov-Galerkin (MLPG) method for the dynamic fracture problem, Engineering Analysis with Boundary Elements, 30, 72-76

25. Liu W.K., Jun S., Zhang Y.F., 1995, Reproducing kernel particle methods, International Journal for Numerical Methods in Engineering, 20, 1081-1106

26. Melenk J.M., Babuska I., 1996, The partition of unity finite element method: Basic theory and applications, Computer Methods in Applied Mechanics and Engineerin, 139, 289-314

27. Mukherjee Y.X., Mukherjee S., 1997, Boundary node method for potential problems, International Journal for Numerical Methods in Engineering, 40, 797-815

28. Nayroles B., Touzot G., Villon P., 1992, Generalizing the finite element method: diffuse approximation and diffuse elements, Computational Mechanics, 10, 307-318

29. PAN X., YUAN H., 2009, Applications of meshless methods for damage computations with finite strains, Modelling and Simulation in Materials Science and Engineering, 17, 4, 045005

30. Shirzadi A., 2014, Solving 2D reaction-diffusion equations with nonlocal boundary conditions by the RBF-MLPG method, Computational Mathematics and Modeling, 25, 4, 521-529

31. Shivanian E., 2013, Analysis of meshless local radial point interpolation (MLRPI) on a nonlinear partial integro-differential equation arising in population dynamics, Engineering Analysis with Boundary Elements, 37, 1693-1702

32. Shivanian E., 2015a, A new spectral meshless radial point interpolation (SMRPI) method: A wellbehaved alternative to the meshless weak forms, Engineering Analysis with Boundary Elements, 54, 1-12

33. Shivanian E., 2015b, Meshless local Petrov-Galerkin (MLPG) method for three-dimensional nonlinear wave equations via moving least squares approximation, Engineering Analysis with Boundary Elements, 50, 249-257

34. Shivanian E., Khodabandehlo H.R., 2014, Meshless local radial point interpolation (MLRPI) on the telegraph equation with purely integral conditions, European Physical Journal Plus, 129, 241-251

35. Singh I.V., Tanaka M., Endo M., 2007, Meshless method for nonlinear heat conduction analysis of nano-composites, Heat and Mass Transfer, 43, 10, 1097-1106

36. Sladek J., Sladek V., Zhang C., TAn C.L., 2006, Evaluation of fracture parameters for crack problems in FGM by a meshless method, Journal of Theoretical and Applied Mechanics, 44, 3, 603-636

37. Tikhonov A.N., Samarski A.A., 1990, Equations of Mathematical Physics, Dover, New York 